Original Paper http://ajol.info/index.php/ijbcs http://indexmedicus.afro.who.int

\title{
Diagnostic du mode d'exploitation et contraintes majeures des vergers fruitiers et autres espèces arboricoles dans la région du Nord Cameroun
}

\author{
Sali BOUROU ${ }^{1}$, Issa ADAMOU ${ }^{2}$, Chantal MADOU ${ }^{1 *}$ et Jean KUATE ${ }^{3}$ \\ ${ }^{I}$ Institut de Recherche Agricole pour le Développement (IRAD) de Garoua \\ Station polyvalente de Recherche de Garoua, B.P. : 415 Garoua, Cameroun. \\ ${ }^{2}$ Ministère de l'Agriculture et du Développement Rural (MINADER), Délégation Régional-Nord, Cameroun. \\ ${ }^{3}$ Institut de Recherche Agricole pour le Développement (IRAD). Direction Générale, \\ B.P. 2123 Yaoundé, Cameroun. \\ *Auteur correspondant ; E-mail : chantal.madou@yahoo.fr
}

\section{REMERCIEMENTS}

Les auteurs remercient le gouvernement Camerounais à travers son budget d'investissement public qui a permis la réalisation de ces travaux.

\section{RESUME}

La disponibilité de vastes terres en friche constitue un immense potentiel de production des arbres fruitiers et autres espèces arboricoles dans la région du Nord, mais l'absence de connaissance sur le fonctionnement des exploitations fruitières a entrainé le désintéressement des producteurs fruitiers. Face à cette situation, une étude a été réalisée dans les quatre départements de la région du Nord à savoir la Bénoué, le Faro, le Mayo-Louti et le Mayo-Rey en vue d'identifier les causes du dépérissement et de proposer des solutions. L'approche méthodologique par des questionnaires adoptés a permis de collecter des données auprès des propriétaires de vergers et suivi d'une descente sur le terrain pour les observations visuelles de ces vergers. Au terme de cette étude 58,2 \% des exploitants sont adultes et $41,8 \%$ ne sont pas scolarisés. On enregistre dix (10) espèces d'arbres fruitiers cultivées, parmi lesquels on a les agrumes et les manguiers en majorité. Tout de même $91 \%$ des producteurs sont propriétaires des terres et $48 \%$ l'ont obtenu par héritage alors que $23 \%$ par achat. La production annuelle est estimée à moins de $500 \mathrm{~kg}$ pour les $52,16 \%$ des producteurs enquêtés et plus de $500 \mathrm{~kg}$ pour les $47,84 \%$. Les principales contraintes rencontrées dans cette activité sont la non disponibilité des terres de qualité, les difficultés d'approvisionnement en eau, le coût élevé des engrais et des produits phytosanitaires et le manque des unités de transformation. Les vergers sont constitués d'arbres fruitiers non améliorés présentant des attaques. De ce fait il est important de vulgariser des variétés améliorées des arbres fruitiers développés par l'IRAD, et leur proposer des appuis techniques pour remédier aux différents problèmes sanitaires.

(c) 2019 International Formulae Group. All rights reserved.

Mots clés : Diagnostic, verger, fruitiers, espèces arboricoles, contrainte, Nord-Cameroun. 


\title{
Diagnosis of the exploitation mode and major constraints of orchards in fruits and other arboreal species in the North Cameroon region
}

\begin{abstract}
The availability of wide fallow lands constitutes a good potential of production of fruits trees and others arboricultural species in Northern region of Cameroon. The lack of knowledge on functioning of exploitation of fruits trees and leads the disinterestedness of producers. Facing this situation, studies was realized in four departments in Northern region: Benoué, Faro, Mayo-Louti and Mayo-Rey at the aim of identify causes of this decay and to propose solutions. Investigations done by using questionnaire helps to collect data from orchard owner following by field trip for visual observation in these orchards. From this study, we figured out that $58.2 \%$ of farmers were adults with $41.8 \%$ unschooled. Among these producers, $76 \%$ practiced combine agriculture with maize as main crop at $38 \%$. Ten species of fruits trees were registered with citrus and mango as the most cultivated. However, $91 \%$ of producers were owner of their lands and $48 \%$ obtained these lands through inheritance and $23 \%$ by buying. The annual production was estimated as at least $500 \mathrm{~kg}$ for producers interviewed and more than $500 \mathrm{~kg}$ for $47.87 \%$ others producers. The principal constraint facing in this activity were the non- availability of good lands, difficulties to get water, the high cost of fertilizers and phytosanitary products and the lack of transformation unity. Orchard was constituted to unimproved fruits trees with several pest attacks. So, it is important to vulgarize improved variety of fruits trees developed by IRAD and provide some technical support to remedy various health problems.
\end{abstract}

(C) 2019 International Formulae Group. All rights reserved.

Keywords: diagnostics, orchard, fruits tree, arboricultural species, constraint, Northern-Cameroon.

\section{INTRODUCTION}

Les conditions agro-écologiques du Nord Cameroun permettent une production fruitière abondante et une disponibilité quasi permanente en fruits sur toutes les périodes de l'année. La disponibilité de vastes terres en friche constitue un immense potentiel d'amélioration du volume de production. Ces conditions agro-écologiques sont favorables à la culture de plusieurs espèces fruitières notamment, le manguier, le goyavier, les agrumes, le papayer, la vigne, le palmier dattier etc. Son climat diversifié permet, pour certaines espèces, une longue période de disponibilité en fruits dans l'année (Temple et al., 2005). Sous l'effet des plans d'ajustement structurel et la nécessité de sécuriser les revenus des ménages dans un contexte de désengagement progressif des Etats, des vergers de grande taille se développent vers les périphéries des villes en croissance offrant ainsi d'importantes opportunités économiques à travers la commercialisation des fruits
(Selmi et Elloumi, 2007) cité par Sougnabe et al. (2010). L'essentiel de la production fruitière provient des plantations paysannes en culture mixte et des plants de case.

Les pertes poste récolte et pendant la récolte sont très importantes à cause des difficultés de commercialisation (Sougnabe et al., 2010) et du sous équipement en matériels de récolte et de conservation. Sur le plan alimentaire, les fruits sont utiles à plus d'un titre : ils peuvent être consommés frais ou après transformation en jus, en confitures et en liqueurs. Les agrumes contiennent par exemple de nombreux sels minéraux et sont riches en vitamines (vitamine C : $39 \mathrm{mg} / 100 \mathrm{~g}$ ). Par ailleurs, ils possèdent des composés permettant une bonne utilisation de la vitamine $\mathrm{C}$ dans l'organisme, que l'on ne retrouve pas dans les polyvitamines de synthèse (Kouebou et al., 2013). Depuis vingtcinq ans, les programmes de recherche de I'IRA (Institut de recherche agronomique) ont permis de mettre au point plusieurs espèces 
d'arbres fruitiers à haut rendement et acclimatés aux différentes zones agroécologiques du pays, c'est ainsi que 140 variétés et porte-greffes d'agrumes, 56 variétés de manguiers et une douzaine de lignées ont été placées en collection vivante en 1985 au verger expérimental de Kismatari. Tout de même la recherche agricole et agroalimentaire dispose de résultats utilisables pour le développement à court terme et à différents stades de la filière (recherche variétale, détermination des conditions optimales de conservation et de transformation). Aujourd'hui, on peut estimer à $80 \%$ le nombre de variétés perdues du fait de manque d'entretien des collections sur l'ensemble du territoire national (IRAD, 2008). Toutefois au verger de Kismatari, ce taux se situe à $30 \%$.

Le seuil de consommation en fruits pour une alimentation équilibrée est loin d'être atteint et cette situation est particulièrement critique pour les savanes d'Afrique Centrale, où règne un climat sec (Woin et al., 2003). Malgré l'aspect porteur de cette filière dans la zone de la Communauté économique et monétaire d'Afrique Centrale (Cemac) et les efforts déployés par la recherche et le développement, l'offre en fruits y reste largement inférieure à la demande. La culture fruitière est peu développée dans les savanes d'Afrique or la zone regorge des ressources naturelles qui peuvent accroitre le niveau de vie de sa population (Woin et Essang ; 2002). Dans une étude de marché des fruits et légumes au Cameroun, Temple (2001) a estimé la production à près de 22792 tonnes tandis que la FAO les estimait à moins de 85 tonnes par an (FAOSTAT, 2011). Ces chiffres montrent que le niveau de la production réelle reste inconnue, mais qu'il est toutefois faible et loin de satisfaire la demande régionale d'agrumes. Le niveau de consommation des fruits se situe bien en dessous des normes fixées par la FAO (70 kg/capita/an) (Sougnabe et al., 2009). Plusieurs facteurs sont liées à l'envol de cette filière dans la région du nord Cameroun, dont les plus importantes sont la maitrise des bonnes pratiques culturales et le contrôle des maladies et ravageurs d'agrumes (Kuaté et al., 2006). L'accès au matériel végétal de qualité est une contrainte peu majeure dans cette région du fait de la présence d'une structure de recherche en charge. Globalement ces dix dernières années ces contraintes ont occasionnés l'abandon des vergers fruitiers et un désintéressement des exploitants. Face à cette situation une étude a été conduite en vue de l'identification des causes du dépérissement des agrumes et autres espèces arboricoles et la mise au point de stratégies de production est nécessaire dans la région du Nord Cameroun. Plus spécifiquement il s'agit de :

$>$ Identifier les différents acteurs engagés dans la filière de production;

> Identifier les espèces fruitières cultivées dans la région du Nord ;

$>$ Identifier les facteurs majeurs qui causent le dépérissement des espèces fruitières

> Estimer le volume de la production fruitière annuelle;

Evaluer la performance du système de commercialisation des produits fruitiers et sa contribution dans le revenu des paysans.

\section{MATERIEL ET METHODES \\ Présentation de la zone d'étude}

L'étude a été réalisée dans la région administrative du nord Cameroun. Elle est la partie centrale de la cuvette de la Bénoué dont le terrain atteint une altitude de $176 \mathrm{~m}$ à Garoua.La zone s'étend entre $8^{\circ}$ et $10^{\circ}$ de latitude Nord et entre $12^{\circ}$ et $16^{\circ}$ de Longitude Est, et est limitée au Nord par la région de l'Extrême-Nord, au Sud par la région de l'Adamaoua, à l'Est par les Républiques du Tchad et de la Centrafrique et à l'Ouest par la République Fédérale du Nigéria (Djoufack Manetsa et al., 2011). La cuvette est orientée d'Est en Ouest mais sur laquelle s'élèvent de petits massifs granitoïdes et volcaniques tels que les Monts de Rolli (2049 m), les Monts Hosséré Vokré (2049 m), le Mont Tcholliré 
(370 m). Au Sud-Ouest, les Monts Atlantika (710 m) surplombent la vallée du Faro. Le climat est tu type tropical soudanien classique, avec des saisons sèches et pluvieuses sensiblement de la même durée. Les pluies sont généralement accompagnées de violents orages en saison de pluie (de mai en octobre) qui font baisser les températures. Au sud de Garoua, une savane boisée aux forêts claires recouvre le sol, mais au nord, elle fait place à une steppe très riche en épineux rabougris et pauvre en espèces feuillues (Balanites aegyptiaca, Ziziphus mauritiana et Acacia senegalensis). Plusieurs cours d'eau situés dans la zone prennent leur source sur le plateau de l'Adamaoua et se jettent ensuite dans la Bénoué, principal fleuve de la cuvette. Le lac Lagdo, dans le nord-ouest du parc national de Bouba Ndjida est un barrage de retenue des eaux utilisées pour la production d'énergie hydro-électrique (Adamou., 2008). La région administrative du nord Cameroun comporte quatre départements à savoir la Bénoué avec chef lieu Garoua, Faro avec chef lieu Poli, Mayo Louti avec chef lieu Guider et Mayo Rey avec chef-lieu Tcholliré (Figure 1).

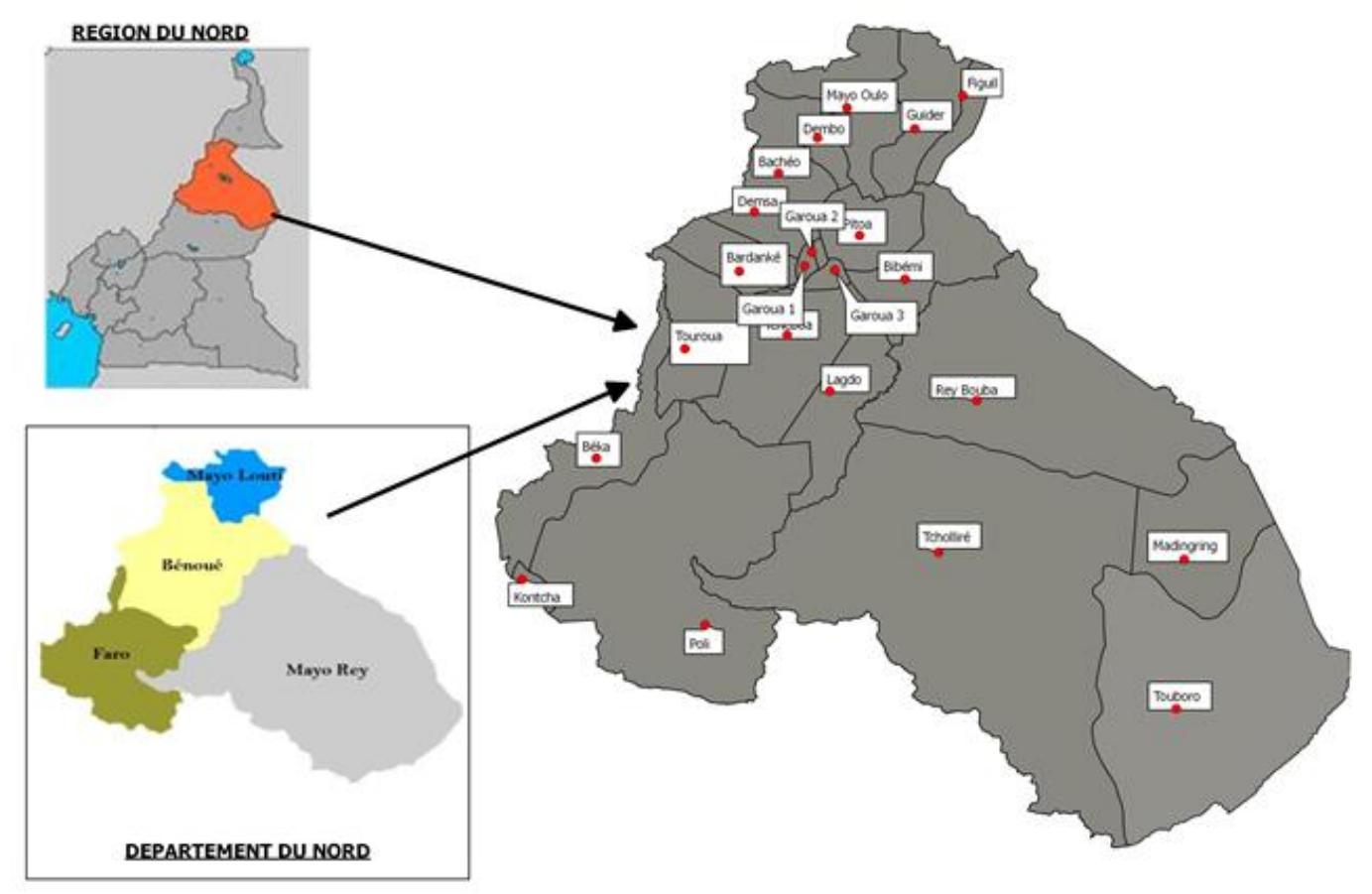

Figure 1 : Carte de la région du Nord-Cameroun et localisation des sites prospectées.

Tableau 1 : Présentation des localités de la zone d'étude.

\begin{tabular}{lll}
\hline Département & Arrondissement & Localité \\
\hline Bénoué & Garoua $1^{\mathrm{er}}$ & Ouro-Labo, Ouro Mal Hammadou \\
& Garoua $2^{\text {eme }}$ & Ouro Aladji, Wafango, Nyakira \\
& Garoua $3^{\text {eme }}$ & Sanguéré Ngal, Kismatari, Bocklé, Mayo-Dadi \\
& Pitoa & Dolla, Bé \\
& Théboa & Djéfatou, Laindé \\
& Bibémi & Bibémi, \\
\hline
\end{tabular}




\begin{tabular}{lll}
\hline Mayo-Louti & Guider & Guider, Lougguérré, Mayo-Loué \\
& Mayo-Oulo & Mandama, Mayo-Oulo, Dourbey \\
Mayo-Rey & Touboro & Touboro, Bogdibo \\
& Tcholliré & Tcholliré, Mayo-Galké \\
Faro & Poli & Poli \\
\hline
\end{tabular}

\section{Méthodologie \\ Zone d'étude}

L'étude a été réalisée entre septembre et décembre 2015 couvrant au total 25 localités réparties dans la Région du Nord. Les localités étudiées que sont les arrondissements et les départements sont présentés dans le Tableau 1.

Dans le cadre de cette étude, deux types de données ont été collectées à savoir : les données primaires et à les données secondaires.

\section{Données primaires}

La collecte des données primaires consistait à administrer les questionnaires aux producteurs fruitiers, effectuer un entretien semi-structuré avec ces producteurs suivi des observations de terrain.

Le questionnaire a pris en compte quelques points essentiels:

- Les questions posées ont été simples, claires et dépourvues de tout terme technique. Le contenu de chaque question a été spécifique et bien défini.

- Les questions ont été posées de manière équilibrée. Une question qui conduit la personne enquêtée à donner une réponse particulière est considérée comme "orientée"; elle a été remplacée par une question formée de façon plus neutre.

- Le temps alloué aux questionnaires a été réduire au maximum

Le questionnaire structuré utilisé a été affiné à partir des résultats de la revue documentaire. Il a ensuite fait l'objet de tests de validation ou enquête pilote auprès de quelques producteurs de Garoua. Ce test a permis de l'améliorer et de le mettre dans un contexte plus réaliste. Les observations directes nous ont permis de collecter des données de terrain. Elle a permis de visualiser et d'apprécier le fonctionnement des exploitations agricoles.

\section{Échantillonnage}

Le but de l'échantillonnage est de fournir suffisamment d'information pour effectuer de l'inférence sur les caractéristiques d'une population. Il a donc pour objectif de sélectionner un échantillon reproduisant le plus fidèlement possible ces caractéristiques. Pour cette étude la méthode d'échantillonnage non-probabilistique a été effectuée. Cette méthode a été choisie du fait que nous ne disposons pas de base de sondage. Car, dans la plus part des méthodes d'échantillonnage aléatoire, il est nécessaire de disposer au départ d'une liste des éléments de population (appelée "base de sondage"). La méthode nonprobalitique, contrairement à l'échantillonnage probabilistique consiste à supposer que la distribution des caractéristiques à l'intérieur de la population est égale. Ainsi, le choix des individus ne se fait pas de manière aléatoire mais raisonnée. Il était question de travailler uniquement avec les producteurs fruitiers.

Les données secondaires ou génériques sont des données issues de la bibliographie ou de travaux de recherche (mémoire, thèse, article ou ouvrage scientifique, rapports).

\section{Analyse des données}

Les données ont été analysées par le logiciel « Statistical Packages for Social Sciences (SPSS) » et le tableur Excel de 
Windows office a été utilisé pour les graphiques et le calcul des moyennes simples. L'analyse a consisté à des statistiques descriptives et des tableaux croisés dynamiques.

\section{RESULTATS \\ Caractéristiques des producteurs fruitiers}

Plus de la moitié des producteurs fruitiers enquêtés $(58,2 \%)$ ont un âge moyen qui varie entre 31 et 60 ans. Cette tranche d'âges démontrent que les enquêtés sont relativement expérimentés, étant donné que les activités agricoles commencent, dès l'âge de 17 ans et que la prise en charge de soi-même se fait autour de 21 ans d'âge. Parmi les producteurs une proportion de $(8,8 \%)$ pratique cette activité depuis leur bas âge à la tranche d'âge comprise entre 0 et 30 ans. Par ailleurs 33\% de ces producteurs donc l'âge est supérieur à 60 ans continuent leurs activités de production fruitière (Figure 2).

\section{Niveau d'étude des producteurs}

$\mathrm{Au}$ plan scolaire, $41,8 \%$ de ces producteurs fruitiers n'ont pas été scolarisés. Parmi les $58,2 \%$ scolarisés, seuls $7,7 \%$ ont atteint le niveau universitaire. Ceci explique le taux élevé des producteurs adolescents qui ont abandonnés l'école au primaire pour se consacrer à l'arboriculture (Figure 3).

\section{Activités des producteurs}

Au terme de l'analyse des activités des producteurs on note que plus de trois quart fruitiers enquêtés (76\%) ont pour activité principale l'agriculture. Toutefois une proportion de $10 \%$ pratique l'agriculture associée à l'élevage, $6 \%$ associe l'agriculture à la pépinière, et $3 \%$ sont des commerçants. Comme partout au Cameroun, dans la région du Nord l'agriculture et l'élevage occupent plus de $90 \%$ de la population. La Figure 4 illustre la répartition des activités des producteurs fruitiers.

\section{Spéculations cultivées}

En plus de la production fruitière, les résultats montrent que ces producteurs sont des adeptes de la diversification agricole. En outre $38 \%$ de cet échantillon cultive le maïs, $13 \%$ le coton qui est une culture de rente dans la zone, $13 \%$ le riz, $11 \%$ le mil, $8 \%$ les tubercules et $5 \%$ les légumineuses. La Figure 5 présente la répartition des spéculations les plus cultivés par les producteurs fruitiers.

\section{Facteurs économiques}

La production des fruits comme toute autre activité est une activité qui génère des revenus. Les revenus issus de cette activité sont plus ou moins importants selon les surfaces exploitées et selon les types d'arbres fruitiers mise en place. Toutefois, la quasi totalité des producteurs enquêtés vendent leurs productions soit $(80 \%)$ et en consomment une partie soit (20\%). Quasiment tous les producteurs fruitiers utilisent les revenus issus de cette vente pour leurs dépenses quotidiennes et le reste est utilisé pour d'autres fins (épargne, investissement etc...).

\section{Propriété foncière}

Il en découle de cette étude qu'environ $91 \%$ des producteurs sont propriétaires des terres utilisées. Parmi ces producteurs $48 \%$ l'ont obtenu par héritage alors que $23 \%$ par achat et le reste l'ont acquis soit par exploitation de parcelle familiale (13\%), soit par don (4\%), ou alors par partage de parcelle (3\%).

\section{Contribution des produits fruitiers au revenu familial}

Après exploitation des données l'on note que les différentes sources de revenu des familles de producteurs proviennent évidemment de la vente des fruits, mais aussi de l'agriculture, de l'élevage, du commerce, de la pêche, du coton et du salaire pour certains producteurs salariés. La vente des fruits est la deuxième source la plus importante après celle de la vente des cultures 
vivrières. Ensuite viennent par ordre d'importance l'élevage et le maraichage.

\section{Contraintes de production}

Les contraintes fréquemment rencontrées par les producteurs fruitiers sont résumées dans le Tableau 2. Il ressort de ce tableau que la contrainte liée à la production est l'utilisation des variétés locales sensibles aux différents ravageurs et maladies. Ceux rencontrés dans la plus part des vergers d'agrumes sont les mouches blanches, les mineuses d'agrumes et les maladies fongiques. La présence des mouches de fruits et de l'anthracnose sont les contraintes observées chez les manguiers. L'on peut également noter le manque d'un programme de traitement phytosanitaire, le manque d'une formulation adaptée d'engrais minéraux, le manque de système d'irrigation adéquat surtout pour les agrumes, la mauvaise pratique de récolte et la mauvaise organisation de la mise en vente et l'écoulement des fruits.

\section{Capital}

La majorité des producteurs ne
possède pas tous les équipements recommandés pour la production des arbres fruitiers. Ils ont en leur procession juste le nécessaire qui leurs permettraient de mettre en place et entretenir leurs cultures. Ces équipements sont: le pulvérisateur, l'atomiseur, la motopompe, la brouette, le pousse-pousse, le sécateur, la machette, la pelle, la pioche et la houe.

\section{Principaux arbres fruitiers cultivés}

Cette étude a montré que dix (10) espèces d'arbres fruitiers sont cultivées dans la Région du Nord. Ces différentes espèces sont exploitées en fonction de leur fréquence de production. Le Tableau 3 présente les différentes espèces d'arbres fruitiers mises en place par les producteurs au Nord Cameroun.

Après analyse, les résultats de ces travaux montrent que le manguier est le plus cultivé dans la zone d'étude, il représente
$27,21 \%$ des vergers. Toutefois les variétés de manguiers les plus utilisées sont le malo et horéwandou qui représentant la moitié de la population de manguier. Les variétés locales paparanda, amelie et algerie qui représentent $11,4 \%$ des plantations. Le reste des variétés de mangues utilisées par les producteurs sont entre autre Julie, IFAC/IRAD, kent et sping. Les goyaviers viennent en deuxième position avec $18 \%$, suivi des citronniers avec $14,39 \%$. La moitié des plants de citronniers est issue des variétés locales et une proportion de 33\% de plant provient de la SODECOTON et le reste des variétés proviennent de l'IRAD. Les orangers constituent $12,43 \%$ des vergers et on y trouve des plants issus des variétés locales et celles améliorées provenant des variétés de l'IRAD 33,3\% (Figure 6). L'étude a montré après analyse que les arbres fruitiers dont l'âge est inférieur à 10 ans représentent la majorité de la population étudiée $(41,81 \%)$, suivi de la tranche de 10 à 20 ans avec 34,63\% et les arbres vieillissants de plus de 20 ans représentant 23,57\% (Figure 7).

Indépendamment des espèces fruitières exploitées la production annuelle par producteur est estimées à plus de $500 \mathrm{~kg}$ pour $47,84 \%$ des producteurs enquêtés. Par ailleurs cette production est estimée à moins de 500 $\mathrm{kg}$ pour une proportion de 52,16\% des producteurs enquêtés. Toutefois cette faible production peut s'expliquer par le caractère jeune de la majorité des exploitations (Figure 8).

\section{Utilisation des intrants agricoles}

Dans notre zone d'étude, les intrants agricoles les plus utilisés sont les pesticides pour $39,8 \%$ de producteurs. Parmi ces produits, l'Optimal est le plus utilisé $(35,5 \%$ des producteurs) suivi de Cypercal utilisé par $32,2 \%$ des producteurs. Le Tableau 4 donne la répartition des pesticides les plus utilisés.

\section{Utilisation de la main d'œuvre}

La plupart des producteurs utilise une main d'œuvre dans leur plantation. Plus de 
$90 \%$ font recours à une main d'œuvre salariée. Cette main œuvre est consacré majoritairement aux travaux de gardiennage, d'entretien et de récolte. On note cependant $9 \%$ qui font recourt à une main d'œuvre familiale. Toutefois la période de recours à la main d'œuvre salariée dépend des besoins du producteur, de ce fait $58 \%$ font recours à la main d'œuvre toute l'année alors que $22 \%$ font appel à cette main d'œuvre en saison de pluie et $9 \%$ en saison sèche.

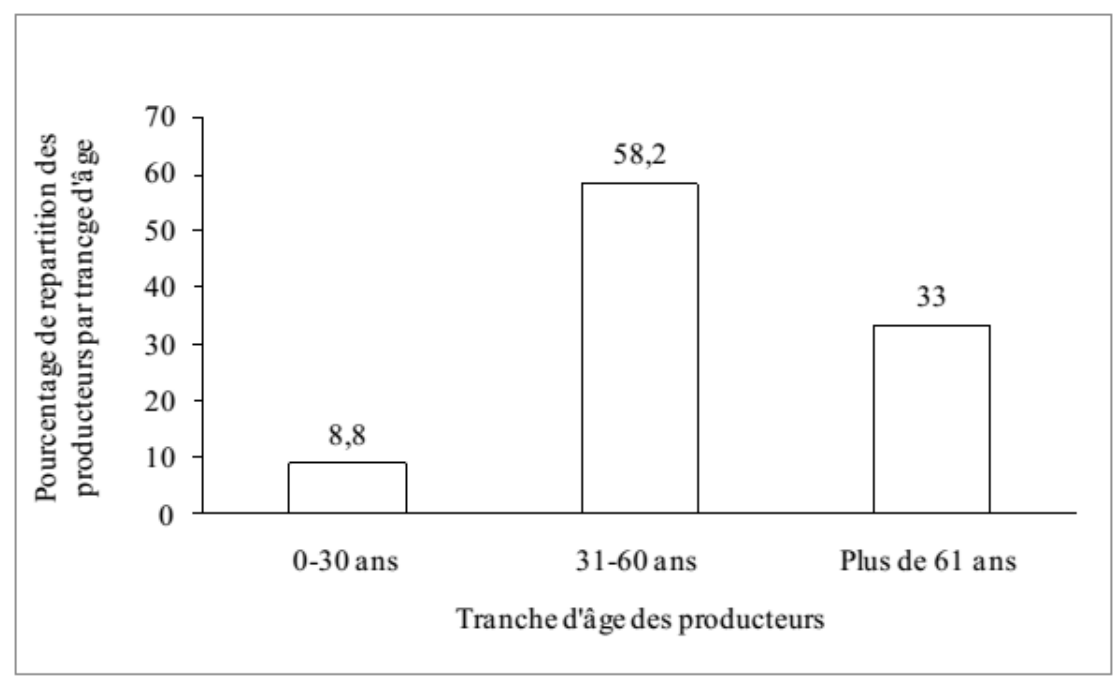

Figure 2 : Répartition des producteurs enquêtés par âge.

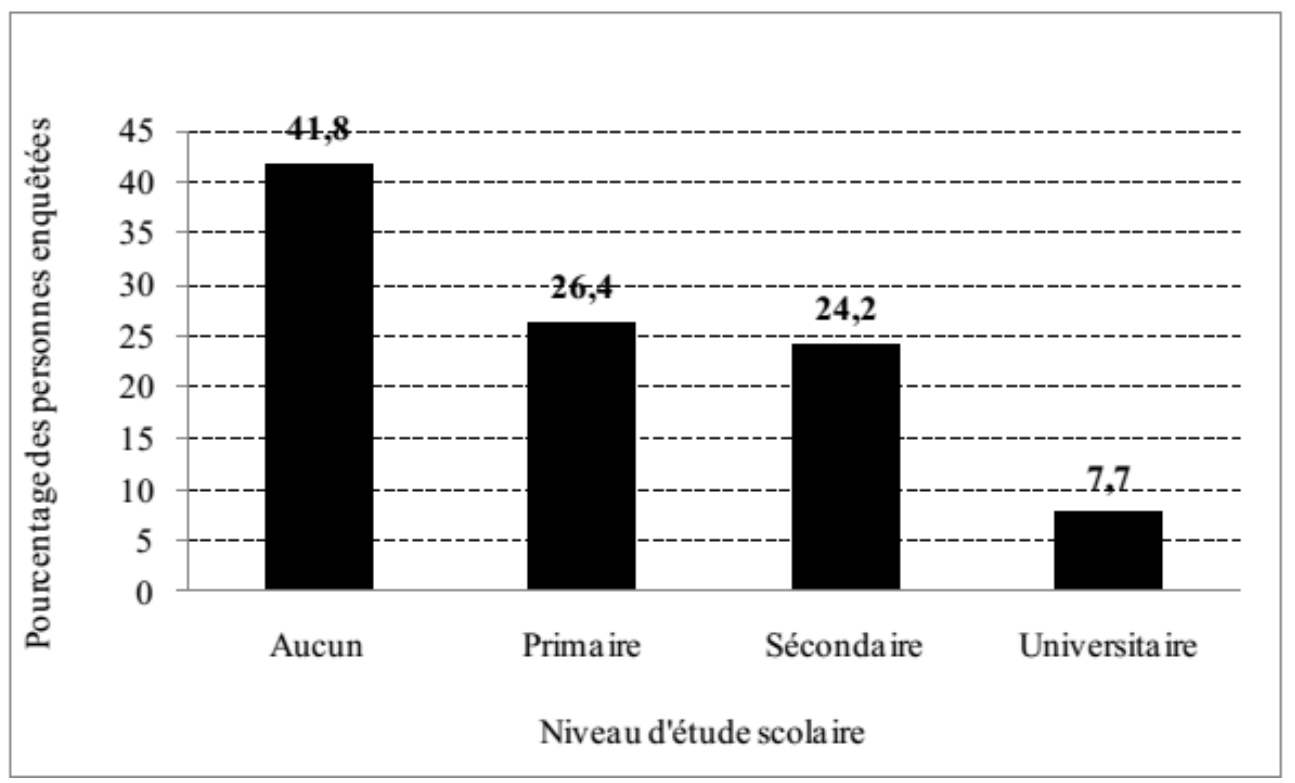

Figure 3 : Répartition des producteurs par niveau d'étude. 


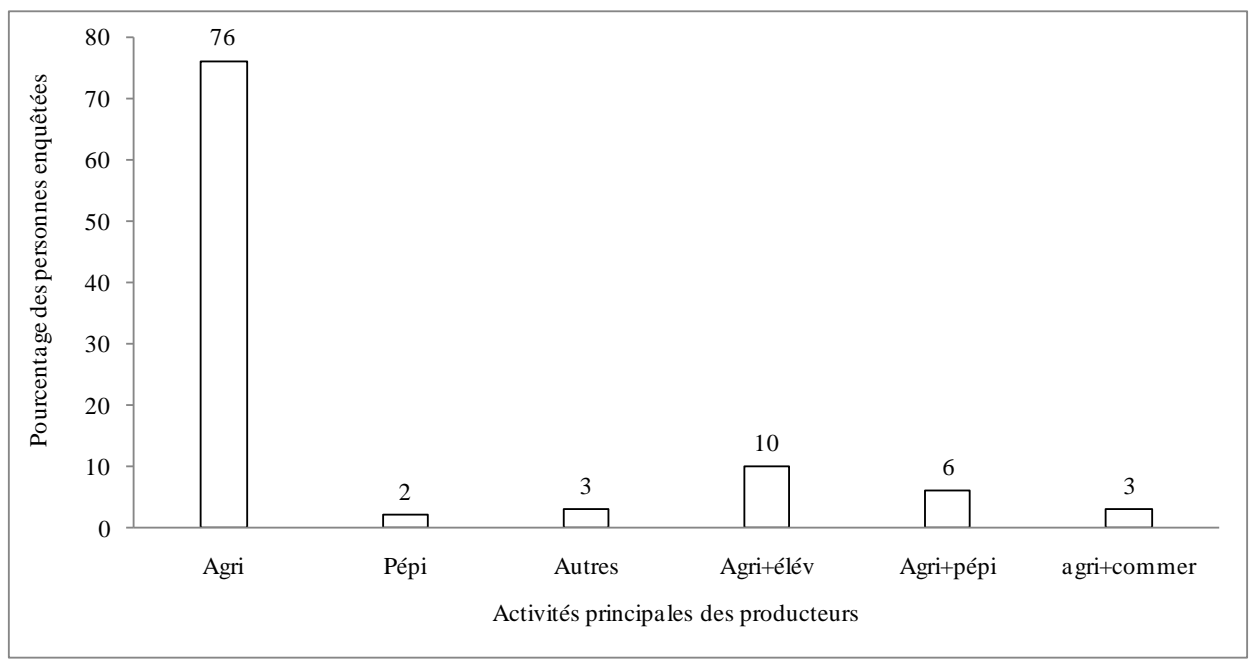

Agri : Agriculture ; Pépi : Pépinière ; Elév : Elevage ; Commer : Commerçant.

Figure 4 : Répartition des producteurs en fonction des activités principales.

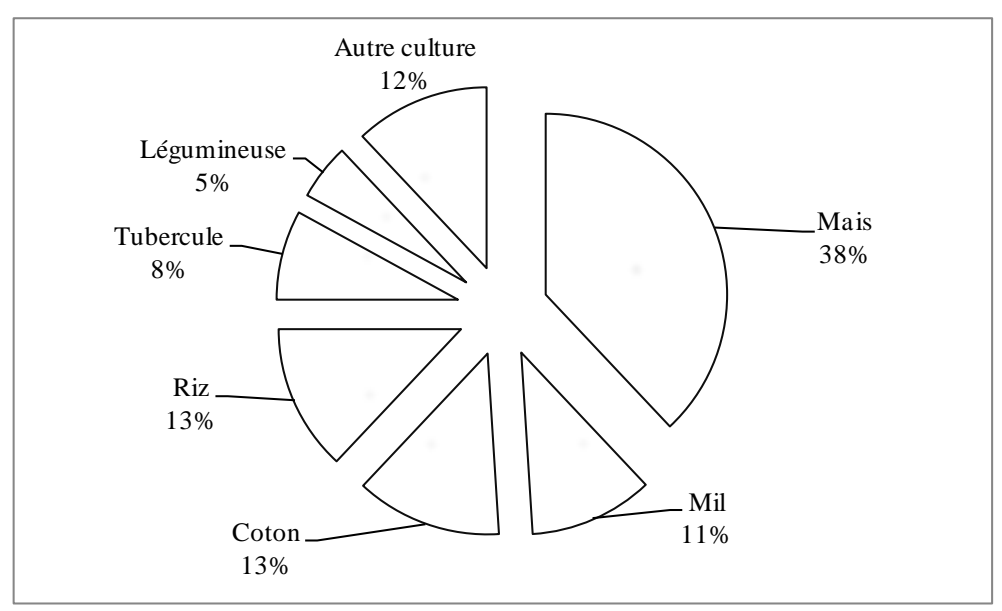

Figure 5 : Répartition des producteurs par spéculation cultivée.

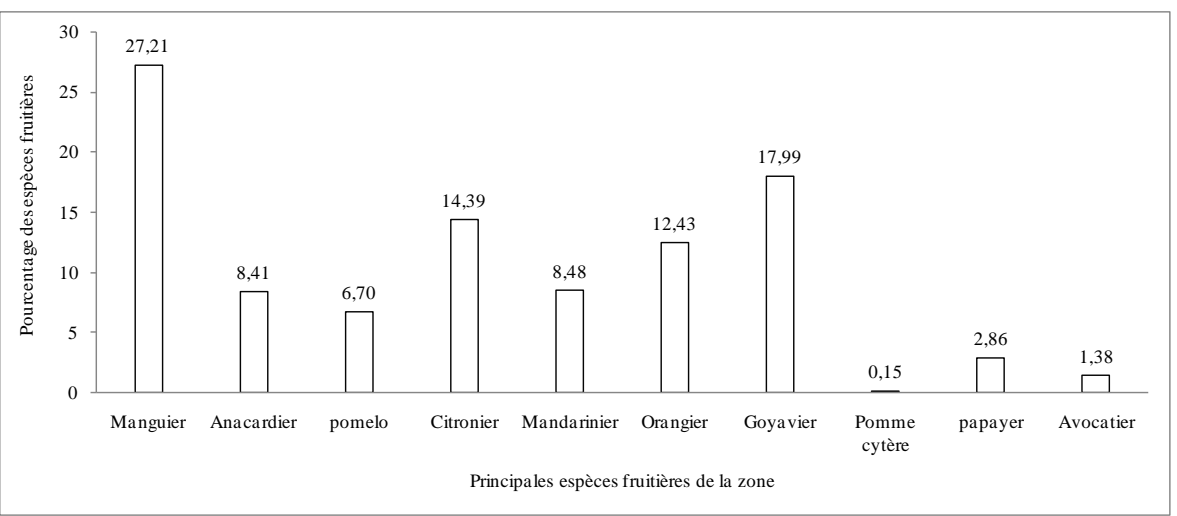

Figure 6: Répartition des espèces fruitières exploitées dans la région du Nord. 


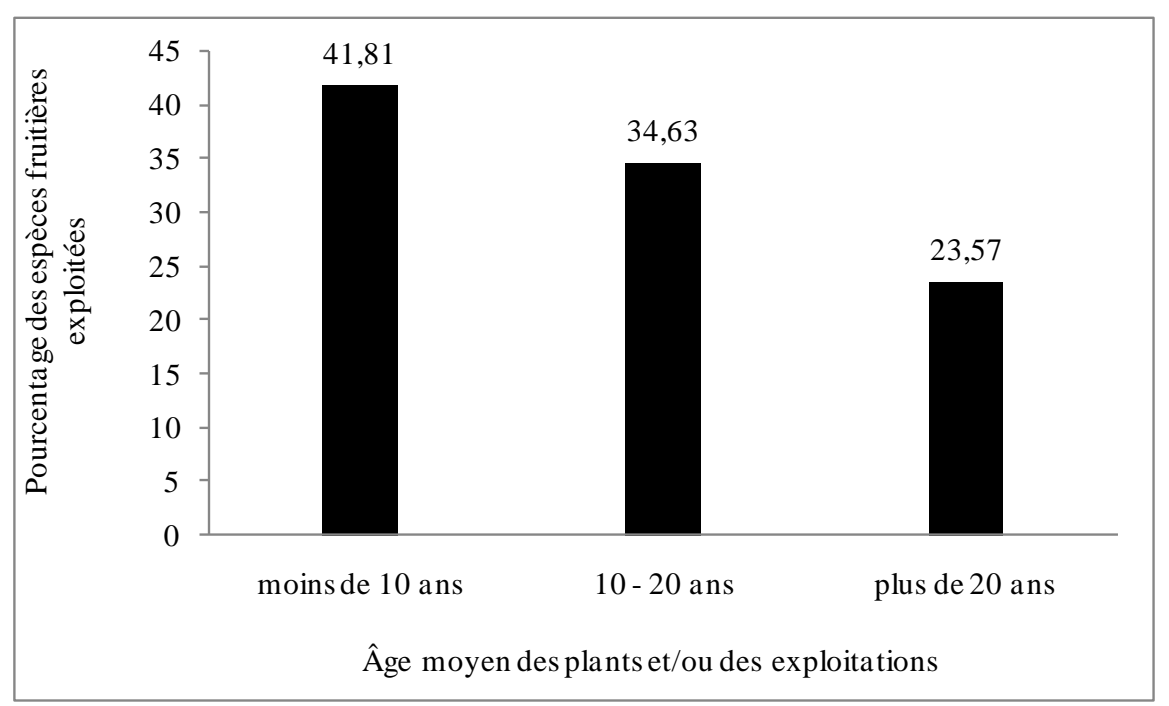

Figure 7 : Age estimé des plants et/ou des exploitations des vergers.

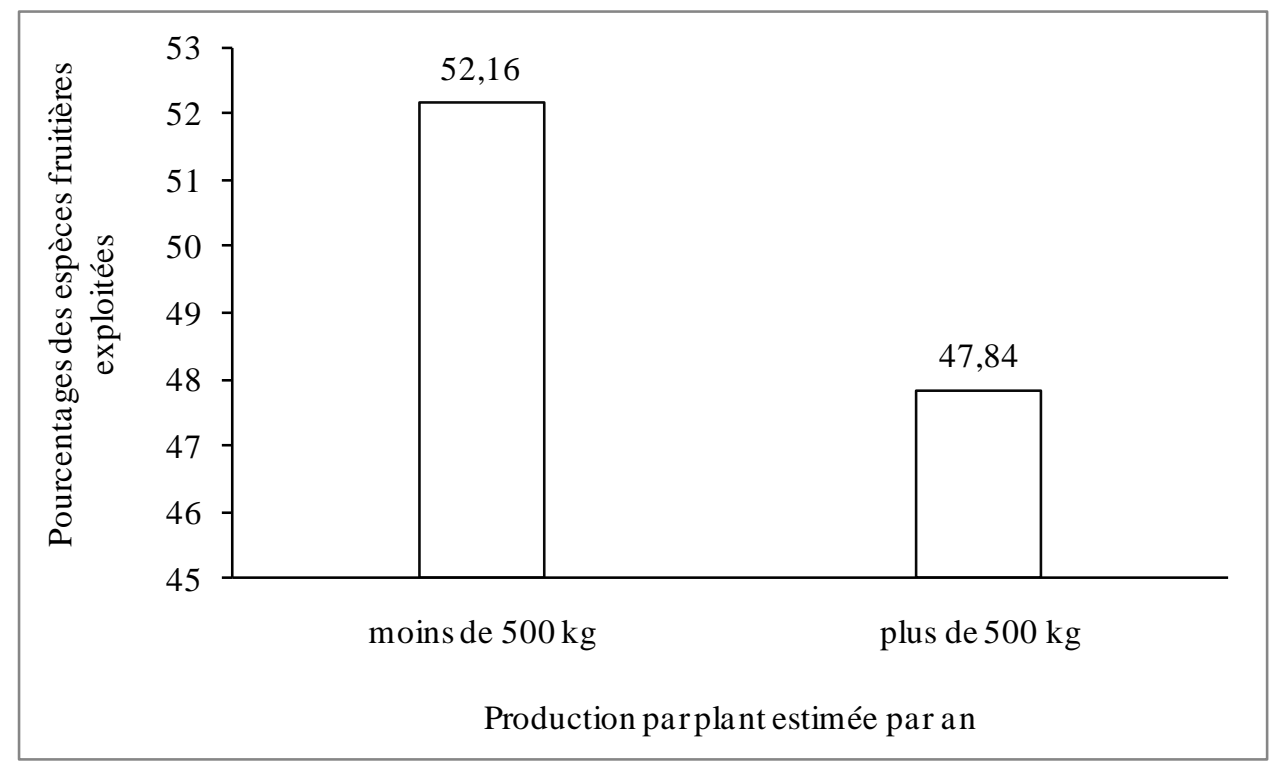

Figure 8: Proportion du volume de production estimée à la récolte.

Tableau 2 : Contraintes couramment rencontré par les producteurs.

\begin{tabular}{lll}
\hline Rubriques & Contraintes \\
\hline $\begin{array}{l}\text { Disponibilités et qualités des } \\
\text { terres }\end{array}$ & $\begin{array}{l}\text { insuffisance des terres aménagées spécifiquement pour } \\
\text { les cultures fruitières } \\
\text { baisse de la fertilité des sols } \\
\end{array}$ & Problème de propriété foncière \\
\hline Disponibilité et qualité $\quad$ de & Absence d'irrigation des vergers en saison sèche
\end{tabular}




\begin{tabular}{ll}
\hline l'eau & Système d'approvisionnement en eau inadapté \\
\hline Disponibilité des intrants & Coût élevé des engrais et produits phytosanitaires \\
$\begin{array}{l}\text { Plants et semences } \\
\text { Produits phytosanitaires }\end{array}$ & $\begin{array}{l}\text { Insuffisance de plants de qualité en quantités } \\
\text { Manque de formulations adaptées de produits de } \\
\text { traitements insecticides }\end{array}$ \\
\hline Technologie de la production & $\begin{array}{l}\text { Manque d'une unité de transformation des fruits } \\
\text { Maladies }\end{array}$ \\
Insectes et ravageumergence de la gommose \\
& $\begin{array}{l}\text { Lutte contre la cochenille et la mouche des fruits } \\
\text { Manque de dispositif de suivi phytosanitaire et du } \\
\text { système d'intervention sur les sites }\end{array}$ \\
\hline Equipement et développement & $\begin{array}{l}\text { Insuffisance de moyens financiers et matériels } \\
\text { Le manque d'organisation des producteurs }\end{array}$ \\
\hline
\end{tabular}

Tableau 3 : différentes espèces mise en place dans la région du Nord.

\begin{tabular}{llll}
\hline Nom & Famille & Nom scientifique & Nom en Fulfulde \\
\hline Manguier & Anacardiceae & Manguifera indica & Mangoro \\
Citronnier & Rutaceae & Citrus limon & LemouLammoudoum \\
Oranger & Rutaceae & Citrus sinensis & Lemou \\
Pomelo & Rutaceae & Citrus maxima & Pamplemousse \\
Anacardier & Anacardiaceae & Anacardium occidentale & Anacardéon \\
Goyavier & Myrtaceae & Psidium guajava & Goyave \\
Papayer & Caricaceæ & Carica papaya & Papaye \\
Avocatier & Lauracees & Persea americana & Phiyo \\
Pomme & Anacardiaceae & Spondias dulcis & - \\
Mandarine & Rutaceae & Citrus reticulata & Mandarine \\
\hline
\end{tabular}

Tableau 4 : Répartition des produits phytosanitaires utilisés.

\begin{tabular}{cccc}
\hline Nom commercial & Matière active & Classe & Pourcentage \\
\hline Optimal 20 SP & Acétamipride 200g/kg & Insecticide & $35,5 \%$ \\
Pacha 25 EC & Lambda-cyhalothrine & Insecticide & $17,5 \%$ \\
& $15 \mathrm{~g} / \mathrm{l}+$ Acétamipride $10 \mathrm{~g} / \mathrm{l}$ & & \\
Cypercal 200 EC & Cyperméthrine $200 \mathrm{~g} / 1$ & Insecticide & $32,2 \%$ \\
Cigogne 360 EC & Cyperméthrine $360 \mathrm{~g} / 1$ & Insecticide & $14,8 \%$ \\
\hline
\end{tabular}

\section{DISCUSSION}

Il ressort de cette étude que la majorité des producteurs sont sous scolarisés, ce résultat est similaire à celui de Sougnabe et al. (2010) où plus de la moitié des exploitants fruitiers est analphabète avec un niveau de formation agricole très faible. Plus de trois quart des producteurs fruitiers enquêtés $(76 \%)$ ont pour activité principale l'agriculture et environ $91 \%$ des producteurs sont propriétaires des terres où ils cultivent. Ces résultats sont semblables à ceux de Sougnabe et al. (2010) qui souligne que la majorité des producteurs a pour activité principale l'agriculture (93\%) et des exploitants (87\%) sont propriétaires des vergers. Cette étude a montré que $(48 \%)$ des producteurs ont obtenu leurs terrains par héritage alors que $(23 \%)$ par 
achat pourtant selon Sougnabe et al. (2010), le mode d'acquisition foncière dominant dans ce bassin est l'achat (84\%). Par ailleurs, une proportion importante de producteurs fruitiers enquêtés $(58,2 \%)$ a un âge moyen variant entre 31 et 60 ans. Ceci montre bien que dans le domaine de production agricole la plupart des enquêtés ont une expérience avérée de près de 15 ans. De même le rapport de l'enquête test sur l'estimation de la production fruitière effectué par l'Observatoire Economique et Statistique d'Afrique Subsaharienne (AFRISTAT) au Mali révèle que la moitié des exploitants ont entre 40 et 60 ans (AFRISTAT, 2001). Ceci indique que l'arboriculture fruitière dans ces zones n'est pas une activité des jeunes.

La majeure partie soit $14,41 \%$ des arbres fruitiers rencontrée dans ces parcelles sont âgées de moins de 10 ans d'âge, avec en association plusieurs autres cultures, avec en majorité du riz. Les cultures associées dépendent de chaque paysan et peuvent varier d'une année à une autre selon les besoins et le désir des producteurs. Au regard des résultats des enquêtés menées dans la préfecture de Tchamba au Togo, pour ce qui est du cas particulier des vergers d'anacarde, la plus importante association pratiquée par les planteurs des localités est celle du coton et de l'anacardier (Sokemawu, 2015). Cette recherche met également en exergue les caractéristiques de diversité biologique végétale des vergers et des espèces arboricoles des producteurs dans la région du Nord Cameroun. Le manguier est l'arbre fruitier le plus exploité des 10 spéculations rencontrées dans la zone d'étude, il représente $(27,21 \%)$ des vergers des producteurs. L'essentiel des producteurs enquêtés consacre la plupart de leur production à la mangue, ce qui est en rapport avec les observations faites dans la zone des Niayes au Sénégal (Oumar, 2008). Cette étude démontre également que l'arbre fruitier et particulièrement le manguier joue un rôle important dans la sécurisation foncière. En effet on constate de cette étude que quel que soit le mode d'acquisition des vergers (achat ou héritage), ces derniers restent un patrimoine familial. Compte tenu de la valeur économique des cultures en places, les paysans ne s'adonnent pas à la vente des terres. (Fatondji, 2012 ; Ayarma, 2015).

Il ressort enfin de cette étude que les contraintes de production des agrumes et manguiers, comme toutes les autres cultures fruitières sont de 3 ordres. L'accès aux intrants agricoles, au crédit, au marché et les problèmes fonciers sont des contraintes d'ordre socio-économiques. La maîtrise des bonnes pratiques culturales et l'accès au matériel végétal de qualité sont des contraintes agronomiques. La présence des maladies et ravageurs constitue une contrainte parasitaire (Kuaté et al., 2006). Cette dernière contrainte est l'une des plus importantes. A côté de ces difficultés on note également le fait que $41,8 \%$ des exploitants de verger n'ont aucun niveau d'instruction, ce qui montre que, comme les autres activités agricoles, l'arboriculture fruitière est pratiquée par des personnes non instruites en majorité. Mais la particularité de l'activité est qu'elle intéresse aussi des personnes d'un certain niveau d'instruction (7,7\%) qui la pratiquent en tant qu'activité secondaire éventuellement. Ce résultat est en accord avec celui AFRISTAT (2001) au mali et en rapport à la répartition des exploitants de verger selon le niveau d'instruction. Face à cette situation, une première piste de réflexion doit être la formation d'animateurs de groupements de producteurs.

\section{Conclusion}

Au terme de cette étude la production fruitière dans la région du Nord est pratiquée par des individus adultes, la majorité ne sont pas scolarisé ou ont le niveau d'éducation scolaire du primaire. Toutefois les $3 / 4$ des producteurs enquêtés cultivent d'autres 
cultures à l'instar du maïs. Tout de même $91 \%$ des producteurs sont propriétaires des terres, et les différentes sources du revenu des familles de producteurs proviennent de l'agriculture, de l'élevage, du commerce, de la pêche, de culture cotonnière, du salaire pour certains producteurs. Aussi La plupart des producteurs ne travaillent pas tout seul dans leur plantation ils font recours à la main d'œuvre. Le résumé de cette étude a montré que dix (10) espèces d'arbres fruitiers sont cultivées dans la Région du Nord et un certains nombres de contraintes de production ont été identifiés. L'étude relève également que les arbres fruitiers dont l'âge est inférieur à 10 ans représentent la majorité de la population étudiée, ce qui pourrait expliquer le faible niveau de production. Il apparaît ainsi important de vulgariser encore au mieux les variétés améliorées de la recherche qui sont plus productives et résistantes. Ces observations doivent conduire à une réflexion approfondie sur l'organisation du marché des fruits, à l'échelle du Cameroun et sur les possibilités d'exportation. De même il est nécessaire de former les producteurs sur les techniques de mise en place et conduite des vergers et sur la transformation des fruits en jus. De ce qui précède, il serait intéressant de développer des programmes de traitement phytosanitaire en fonction des ravageurs et des maladies recensés. Un soutien financier et un renforcement de capacité des producteurs fruitiers sont aussi nécessaires.

\section{CONFLIT D'INTERETS}

Les auteurs déclarent qu'ils n'ont aucun conflit d'intérêt.

\section{CONTRIBUTIONS DES AUTEURS}

$\mathrm{SB}$ et $\mathrm{CM}$ ont contribué à la rédaction de l'article et à l'établissement du protocole de collecte. JK et IA ont contribué à la collecte, à l'analyse des données et à l'enrichissement l'article.

\section{REFERENCES}

Achancho V. 2013. Revue et analyse des stratégies nationales d'investissements et des politiques agricoles en Afrique du Centre: Cas du Cameroun: Reconstruire le potentiel alimentaire de l'Afrique de l'Ouest, A. Elbehri (éd.) FAO/FIDA. 48 p.

Adamou I. 2008. Rapport de stage de pré insertion professionnelle; Université de Dschang $53 \mathrm{p}$.

AFRISTAT. 2001. Enquête test sur l'estimation de la production fruitière au Mali. Rapport final, Série Méthodes ${ }^{\circ} 5$ Mai.

Ayarma A. 2015. L'insécurité foncière comme obstacle au développement et à la conservation de la biodiversité en pays Kpélé au Sud-Ouest du Togo. Mémoire de Master Recherche, Science de l'Homme et de la Société (SHS), Société-Environnement-Aménagement, Département de Géographie, Université de Lomé, Lomé, 111 p.

Fatodji K. 2012. Impacts socio-économiques de la dynamique foncière dans les espaces ruraux autour des villes en Afrique subsaharienne : approche bibliographique. Mémoire de DEA, Université de Lomé, Lomé, 117 p.

Kouebou C, Fadi Goygoy, Bourou S, Kosga Djakissam P, Layla H, Zenabou G, Barbi M, Vunyingah M, Woin N. 2013. Biodiversité et valeur alimentaire des fruits au Cameroun : observations préliminaires dans le Département de la Bénoué (Région du Nord). Journal of Applied Biosciences, 69: 5510 - 5522

Kuate J, Bella-Manga, Damesse F, Kouodiekong L, Ndindeng SA, David O. 2006. Enquête diagnostic sur les fruitiers dans les exploitations familiales agricoles en zone humide du Cameroun. Fruits, 61(6) : 373-387.

Manetsa V. 2011. Etude multi-échelles des précipitations et du couvert végétal au 
Cameroun : analyses spatiales, tendances temporelles, facteurs climatiques et anthropiques de variabilité du NDVI. Sciences de la Terre. Université de Bourgogne.

Oumar D. 2008. L'amélioration des revenus et de la sécurité alimentaire des petits exploitants en Afrique de l'Ouest et en Afrique Centrale par l'exportation de produits tropicaux biologiques et $\mathrm{du}$ commerce équitable» Rapport de l'évaluation d'impact du projet, la mangue biologique dans la zone des Niayes au Sénégal. Ecole Nationale Supérieure d'Agriculture de Thiès. FAO, GCP/RAF/404/GER.

Sokemawu K. 2015. Le développement de la filière anacarde dans la préfecture de Tchamba au Togo : vers une nouvelle stratégie paysanne de diversification des revenus agricoles. Revue de Géographie de l'Université de Ouagadougou, 2(04).

Sougnabe SP, Woin N, Lyannaz J P, Rey JY, Sali B. 2010. Caractérisation des bassins et des systèmes de production fruitière dans les savanes d'Afrique centrale. Acte du Colloque des Savanes africaines en développement : innover pour durer, Garoua, Cameroun. Cirad, 10 p.

Tsafack NG. 2004. Rapport de l'étude socioéconomique du système de commercialisation des amandes de mangues sauvages (irvingia spp) dans l'arrondissement de Ngoulemakong (sudCameroun) 1-12 p.

Woin N, Essang T. 2003. Arboriculture fruitière : problématique, enjeux et rôles dans le développement économique des savanes d'Afrique centrale. $25 \mathrm{p}$. 